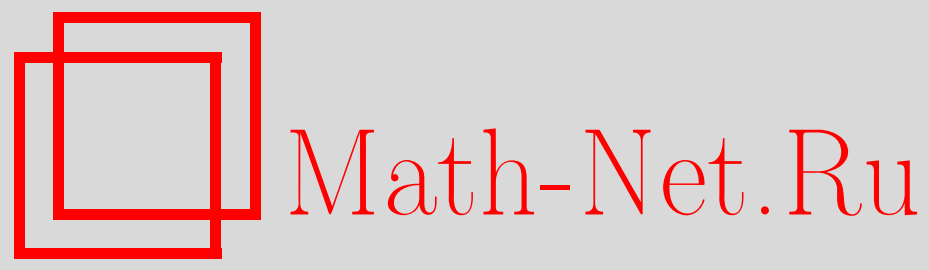

Г. И. Просветов, Минимальная система определяющих соотношений для некоторых супералгебр Ли, Функи. анализ и его прил., 1997, том 31, выпуск 1, 89-92

DOI: https://doi.org/10.4213/faa453

Использование Общероссийского математического портала MathNet.Ru подразумевает, что вы прочитали и согласны с пользовательским соглашением http://www . mathnet.ru/rus/agreement

Параметры загрузки:

IP : 54.237 .206 .68

26 апреля 2023 г., 14:41:28

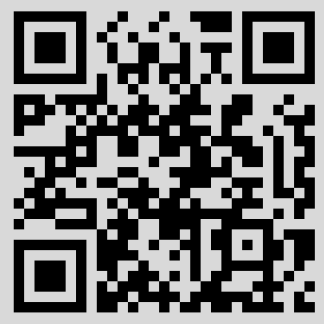


ТЕОРЕМА 3. Пусть начальнье даннье для (1) удовлетворяют условию $\left\|C_{n}(0)\right\| \leqslant K \quad$ u $C_{n}(0)$ обратимьи, $n \in \mathbb{Z}$. Тогда существует $\delta=\delta(K)>0$, такое, что при $t \in[0, T)$ решение задачи Коши для (1) существует, единственно и представимо в виде ряда

$$
C_{n}(t)=\sum_{p=0}^{\infty} \frac{C_{n p} t^{p}}{p !}, \quad n \in \mathbb{Z} .
$$

Коэффициентьи $C_{n р}$ определяются следуюшим образом:

(i) строится конечная матрица $L(n, p, 0)=\left(L(n, p, 0)_{i j}\right)_{i, j=0}^{q(2 p+1)}$ с ненулевыми элементами

$$
L(n, p, 0)_{i, i-q}=C_{n-p q+i-q}(0), \quad L(n, p, 0)_{i, i+1}=E, \quad i=0, \ldots, q(2 p+1) ;
$$

(ii) определяется моментная последовательность $S\left(\mathfrak{M}_{p}(\lambda, 0)\right)=\left(S_{k, p}^{1}(0)\right.$, $\left.\ldots, S_{k, p}^{q}(0)\right)_{k=0}^{\infty}$ по формуле $S_{k, p}^{i}=\left(L^{k}(n, p, 0)\right)_{0, i-1}, i=1, \ldots, q$;

(iii) при помощи формул (8) теоремь 2 и (6) теоремь 1 строится последовательность $\left(S_{k, p}^{1, l}(t), \ldots, S_{k, p}^{q, l}(t)\right)_{k=0}^{\infty}=S^{l}\left(\mathfrak{M}_{p}(\lambda, t)\right), l \in \mathbb{Z}_{+} ;$

(iv) вьчисляются $C_{n p}$ по формулам

$$
C_{n p}=\left.\frac{d^{p}}{d t^{p}}\left(S_{q+1, p}^{1, q p}(t)\right)\right|_{t=0} .
$$

Автор благодарен А. Г. Костюченко за внимание к работе.

$$
\text { ЛитЕРАТУРА }
$$

1. Kac M., van Moerbeke P. Adv. Math., 16, 160-169 (1975). 2. Berezansky Yu. M. Rep. Math. Phys., 24, No. 1, 21-47 (1986). 3. Богоявленский О. И. Изв. АН СССР, cер. матем., 51, №6, 1123-1142 (1987). 4. Itoh I. Progr. Theoret. Phys., 7, No. 3, 507-510 (1987). 5. Гехтман М. И. Функц. анализ и его прил., 24, вып. 3, 72-73 (1990). 6. Гехтман М. И. Функц. анализ и его прил., 25, вып. 3, 82-84 (1991).

Институт системных исследований РАН

Поступило в редакцию 22 ноября 1995 г.

УДК 519.46

\section{Минимальная система определяющих соотношений для некоторых супералгебр Ли}

(C) 1997. Г.И. Просветов

В данной работе найдены минимальные системы определяюших соотношений (т.е. минимальные системы соотношений между образующими) для некоторых супералгебр Ли.

Для алгебры Каца-Муди $L(A)$ с обобщенной $n \times n$-матрицей Картана $A=$ $\left(a_{i j}\right)$ и образующими Шевалле $e_{i}, f_{i}(i=1, \ldots, n)$ имеется треугольное разложение $L(A)=L_{-} \oplus L_{0} \oplus L_{+}$. Здесь $L_{+}$(соответственно $\left.L_{-}\right)$обозначает подалгебру в $L(A)$, порожденную элементами $e_{1}, \ldots, e_{n}\left(\right.$ соответственно $\left.f_{1}, \ldots, f_{n}\right)$, а 
$L_{0}$ - подалгебра Картана. Определяющая система для $L_{+}$(соответственно $L_{-}$) — это $\left(\operatorname{ad} e_{i}\right)^{1-a_{i j}} e_{j}=0$ (соответственно $\left.\left(\operatorname{ad} f_{i}\right)^{1-a_{i j}} f_{j}=0\right), i \neq j$ (см. [1]).

Известно, что подалгебра $\bigoplus_{k=2}^{\infty} \mathbb{C}[p] q^{k}$ алгебры Пуассона $P=\mathbb{C}[[p, q]]$ имеет образуюшие $p^{k} q^{2}, k=0,1, \ldots$, и минимальную систему определяющих соотношений степеней 2 и 3 . Следует напомнить, что алгебра Ли полиномиальных гамильтоновых векторных полей на плоскости есть факторалгебра алгебры $P$ по ее центру. Через $\mathbb{C}[p]$ обозначено кольцо многочленов от $p$. Рассмотрим вопрос о минимальной системе определяюших соотношений для супералгебры Ли

$$
L=\mathbb{C}[p] q \xi \oplus \mathbb{C}[p] q^{2} \oplus \mathbb{C}[p] q^{2} \xi \oplus \mathbb{C}[p] q^{3} \oplus \mathbb{C}[p] q^{3} \xi \oplus \cdots .
$$

Здесь $\xi^{2}=0$. При помощи отображения

$$
H(p, q, \xi) \mapsto \frac{\partial H}{\partial q} \frac{\partial}{\partial p}-\frac{\partial H}{\partial p} \frac{\partial}{\partial q}-\frac{\partial H}{\partial \xi} \frac{\partial}{\partial \xi}
$$

устанавливается изоморфизм между супералгеброй $L$ и подсупералгеброй супералгебры Ли полиномиальных гамильтоновых векторных полей, сохраняющих 2-форму $d p \wedge d q+(d \xi)^{2}$. Коммутатор в $L$ задается формулой

$$
\left[H_{1}(p, q, \xi), H_{2}(p, q, \xi)\right]=\frac{\partial H_{1}}{\partial p} \frac{\partial H_{2}}{\partial q}-\frac{\partial H_{2}}{\partial p} \frac{\partial H_{1}}{\partial q}+\frac{\partial H_{1}}{\partial \xi} \frac{\partial H_{2}}{\partial \xi} .
$$

Далее через $W_{1}$ мы обозначаем алгебру Ли полиномиальных векторных полей на прямой, через $F_{\mu}$ - пространство выражений вида $f(x) d x^{-\mu}$, где $f(x)-$ многочлен, а через $[n]$ — целую часть числа. Супералгебра $L$ является градуированной:

$$
\operatorname{deg} e_{k}^{(\lambda)}=\lambda, \quad e_{k}^{(\lambda)}=p^{k} q^{\mu} \xi^{m}, \quad \mu=[\lambda / 2]+1, k=0,1, \ldots, m=\lambda \bmod 2 .
$$

Мы можем изобразить элементы нашей супералгебры в виде целочисленной решетки на плоскости, в которой элементам $e_{k}^{(\lambda)}$ соответствует точка с координатами $(\lambda, \mu-k)$. Как $\mathbb{C}[p] q\left(\simeq W_{1}\right)$-модуль $L$ изоморфна

$$
F_{1} \oplus F_{2} \oplus F_{2} \oplus F_{3} \oplus F_{3} \oplus \cdots,
$$

где элементам степени $\lambda$ супералгебры $L$ соответствует $F_{\mu}, \mu=[\lambda / 2]+1$. Образующими для $L$ являются элементы $e_{k}^{(1)}, k=0,1, \ldots$.

Теорема. Минимальная система определяюших соотношений для $L$ содержит только соотношения степеней $2,4,5$, причем соотношения степени 2 составляют ядро отображения $S^{2} F_{1} \rightarrow F_{2}$, а соотношения степеней 4 и 5 определяют соответственно $F_{1}$ и $F_{4}$ (как $W_{1}$-модули). А именно, соотношения степени 2

$$
\left[e_{0}^{(1)}, e_{n}^{(1)}\right]=\left[e_{\alpha}^{(1)}, e_{\beta}^{(1)}\right], \quad \alpha+\beta=n, \alpha \neq 0, \alpha \leqslant \beta, n=1,2, \ldots,
$$

соответствуют ядру отображения $S^{2} F_{1} \rightarrow F_{2}$, соотношения степени 4 $k\left[e_{0}^{(1)},\left[e_{0}^{(1)},\left[e_{1}^{(1)}, e_{k}^{(1)}\right]\right]\right]-(k+1)\left[e_{1}^{(1)},\left[e_{0}^{(1)},\left[e_{0}^{(1)}, e_{k}^{(1)}\right]\right]\right]=0, \quad k=2,4,5,6, \ldots$, соответствуют $W_{1}$-модулю $F_{1}$, а соотношения степени 5 $(m-3)\left[e_{0}^{(1)},\left[e_{0}^{(1)},\left[e_{0}^{(1)},\left[e_{1}^{(1)}, e_{m+1}^{(1)}\right]\right]\right]\right]-(m+2)\left[e_{1}^{(1)},\left[e_{0}^{(1)},\left[e_{0}^{(1)},\left[e_{0}^{(1)}, e_{m+1}^{(1)}\right]\right]\right]\right]=0$, 
$m=-1,0,1, \ldots$, соответствуют $W_{1}$-модулю $F_{4}$.

ДокАЗАТЕльство. Случай соотношений степени 2 ясен.

Для Элементов степени $\lambda_{1}, \lambda_{2}, \lambda_{3}$ имеем

$$
\begin{gathered}
\partial: F_{\mu_{1}} \otimes F_{\mu_{2}} \otimes F_{\mu_{3}} \rightarrow F_{\mu_{1}} \otimes F_{\nu_{2}}+F_{\mu_{2}} \otimes F_{\nu_{3}}+F_{\mu_{3}} \otimes F_{\nu_{1}}, \\
\mu_{i}=\left[\lambda_{i} / 2\right]+1, \quad \nu_{i}=\left[\left(\lambda_{i}+\lambda_{i+1}\right) / 2\right]+1, \quad i=1,2,3, \lambda_{4}=\lambda_{1}
\end{gathered}
$$

(где $\partial-$ дифференциал комплекса). Напомним (см. [2]), что композиционный ряд модуля $F_{\lambda} \otimes F_{\mu}-$ это

$$
F_{\lambda+\mu}, F_{\lambda+\mu-1}, F_{\lambda+\mu-2}, \ldots
$$

т.е. в $F_{\lambda} \otimes F_{\mu}$ имеется фильтрация

$$
F_{\lambda} \otimes F_{\mu}=\Phi_{0} \supset \Phi_{1} \supset \Phi_{2} \supset \cdots, \quad \Phi_{i} / \Phi_{i+1} \simeq F_{\lambda+\mu-i} .
$$

Сравнивая композиционные ряды в левом и правом модулях отображения $S^{3} F_{1} \rightarrow F_{1} \otimes F_{2}$, убеждаемся, что нет соотношений степени 3 .

Отождествим $F_{\mu}$ с $R_{1}=\mathbb{C}[x]$, поставив в соответствие полю $f(x) d x^{-\mu}$ полином $f(x)$. При этом $F_{\mu_{1}} \otimes \cdots \otimes F_{\mu_{n}}$ отождествляется с $R_{n}$ - кольцом многочленов от $n$ переменных с комплексными коэффициентами. Супералгебра $L$ имеет $(\mathbb{Z} \oplus \mathbb{Z})$-градуировку: $\operatorname{deg} e_{k}^{(\lambda)}=(\lambda, \mu-k), \mu=[\lambda / 2]+1$. Благодаря этому градуировку получают ее гомологии. При $\lambda_{1}=\lambda_{2}=1, \lambda_{3}=2 s$ (соответственно $\left.\lambda_{3}=2 s+1\right), s=1,2, \ldots,(1)$ запишется в виде $S^{2} F_{1} \otimes F_{s+1} \rightarrow$ $F_{1} \otimes F_{s+1}+F_{2} \otimes F_{s+1}\left(\right.$ соответственно $\left.S^{2} F_{1} \otimes F_{s+1} \rightarrow F_{1} \otimes F_{s+2}+F_{2} \otimes F_{s+1}\right)$, причем оператор

$$
A_{1}=\left.\left((s+1) \frac{\partial}{\partial x_{2}}-\frac{\partial}{\partial y}\right)\right|_{u=x_{2}=y, v=x_{1}}
$$

(соответственно $A_{2}=\left.\right|_{u=x_{2}=y, v=x_{1}}$ ) отображает левую часть на первое слагаемое правой части, а оператор $B=\left.\right|_{u=x_{1}=x_{2}, v=y}$ - на второе. Оператор $B$ переводит в нуль функции вида

$f\left(x_{1}, x_{2}, y\right)\left(x_{1}-x_{2}\right)^{m}\left(\left(x_{1}-y\right)^{n}+\left(x_{2}-y\right)^{n}\right), \quad n=0,1, \ldots, m=2,3, \ldots$.

Здесь $f$ - симметричный по $x_{1}, x_{2}$ многочлен. Подействуем на эти функции оператором $A_{1}$ (соответственно $A_{2}$ ). Под действием оператора $A_{1}$ (coответственно $\left.A_{2}\right)$ мы получаем при $s=2,3, \ldots($ соответственно $1,2, \ldots)$ из $S^{2} F_{1} \otimes F_{s+1}$ все члены композиционного ряда $F_{s+2}, F_{s+1}, F_{s}, \ldots$ (соответственно $\left.F_{s+3}, F_{s+2}, F_{s+1}, \ldots\right)$ модуля $F_{1} \otimes F_{s+1}\left(\right.$ соответственно $\left.F_{1} \otimes F_{s+2}\right)$, за исключением $F_{s+2}$ (соответственно $\left.F_{s+3}, F_{s+2}\right)$. При $s=1$ под действием $A_{1}$ мы не можем попасть в $F_{3}$ и $F_{1}$. Вычисляя двумерные гомологии степеней $(2(s+1), s+2), s=2,3, \ldots,(4,3-k), k=0,1, \ldots,(3+2 t, t+3)$, $(3+2 t, t+2), t=2,3, \ldots,(5,3-m), m=-1,0,1, \ldots$, мы получаем, что нетривиальными являются лишь пространства $H_{2}^{(4,3-k)}(L), k=2,4,5,6, \ldots$, $H_{2}^{(5,3-m)}(L), m=-1,0,1, \ldots$ Эти пространства одномерны, и представляющие циклы равны $e_{k}^{(3)} e_{0}^{(1)}-e_{k-1}^{(3)} e_{1}^{(1)},(m-3) e_{m+1}^{(4)} \otimes e_{0}^{(1)}-(m+1) e_{m}^{(4)} \otimes e_{1}^{(1)}$, откуда получается искомая система соотношений. Теорема доказана.

Рассмотрим эту же задачу для еше одного класса подсупералгебр. Мы получим аналог серровских соотношений. Напомним, что супералгебра Ли $s l(n, n)$ 
состоит из блочных матриц $\left(\begin{array}{ll}A & B \\ C & D\end{array}\right)$, удовлетворяющих условию $\operatorname{tr} A=\operatorname{tr} D$ (подробности см. в [3]). Обозначим через $\left(a_{i j}, b_{i j}, c_{i j}, d_{i j}\right)$ матрицу, у которой на пересечении строки $i$ и столбца $j$ блоков $A, B, C$ и $D$ стоят единицы, а на остальных местах находятся нули. Рассмотрим максимальные нильпотентные подсупералгебры $L^{(1)}$ супералгебр Ли $s l(n+1, n), s l(n, n), n \geqslant 2$, с образуюшими

1) $b_{k k}, c_{1 n}, c_{j+1, j}, k=1, \ldots, n, j=1, \ldots, n-2$, для $\operatorname{sl}(n, n)$,

2) $b_{k+1, k}, c_{1, n+1}, c_{21}, c_{j j}, k=1, \ldots, n, j=3, \ldots, n$, для $\operatorname{sl}(n+1, n)$. Тогда имеет место разложение

$$
\operatorname{sl}(n, n) \text { (или } \operatorname{sl}(n+1, n))=L^{(1)} \oplus L^{(0)} \oplus L^{(2)},
$$

где $L^{(0)}$ - картановская подсупералгебра, а образующие для $L^{(2)}$ симметричны образующим для $L^{(1)}$ относительно главной диагонали. Супералгебра $L^{(1)}$ градуирована. Благодаря этому градуировку получают ее гомологии. Вычисляя двумерные гомологии, получаем, что нетривиальными являются лишь $H_{2}^{(2)}\left(L^{(1)}\right)$, $H_{2}^{(4)}\left(L^{(1)}\right)$. Найдя представляющие циклы, доказываем

ПредлОЖЕНИЕ. Минимальная система определяющих соотношений супералгебры $L^{(1)}$ состоит только из соотношений степеней 2 и 4 , причем соотношения степени 2 имеют вид

$$
\left[b_{i_{1} j_{1}}, b_{i_{2} j_{2}}\right]=0, \quad\left[c_{i_{1} j_{1}}, c_{i_{2} j_{2}}\right]=0, \quad\left[b_{\alpha \beta}, c_{m r}\right]=0, \quad \alpha \neq r, \beta \neq m,
$$

а соотношения степени 4 имеют вид

$$
\begin{array}{rlrl}
{\left[\left[\left[b_{11}, c_{1 n}\right], b_{n n}\right], c_{1 n}\right]} & =0, & & \\
{\left[\left[\left[b_{j+1, j+1}, c_{j+1, j}\right], b_{j j}\right], c_{j+1, j}\right]} & =0, & & j=1, \ldots, n-2, \\
{\left[b_{i i},\left[c_{i+1, i},\left[b_{i i}, c_{i, i-1}\right]\right]\right]} & =0, & i=2, \ldots, n-2,
\end{array}
$$

для случая $\operatorname{sl}(n, n)$ и вид

$$
\begin{array}{rlrl}
{\left[\left[\left[b_{21}, c_{1, n+1}\right], b_{n+1, n}\right], c_{1, n+1}\right]} & =0, & \\
{\left[\left[\left[b_{t+1, t}, c_{t t}\right], b_{t, t-1}\right], c_{t t}\right]} & =0, & & t=3, \ldots, n, \\
{\left[b_{s+1, s},\left[c_{s+1, s+1},\left[b_{s+1, s}, c_{s s}\right]\right]\right]} & =0, & & s=3, \ldots, n-1,
\end{array}
$$

для случая $\operatorname{sl}(n+1, n)$.

Автор признателен Б. Л. Фейгину за постановку задачи и помощь в работе.

$$
\text { ЛитеРАТУРА }
$$

1. Каи В. Г. Бесконечномерные алгебры Ли. Мир, М., 1993. 2. Фукс Д. Б. Когомологии бесконечномерных алгебр Ли. Наука, М., 1984. 3. Kac V. G. Adv. Math., 26, No. 1 (1977). 